\title{
Machining conditions-based preventive maintenance
}

\section{Selim \& Akturk*\#Sinan Gurel}

To cite this article: M. Selim \& Akturk*\#Sinan Gurel (2007) Machining conditions-based preventive maintenance, International Journal of Production Research, 45:8, 1725-1743, DOI: 10.1080/00207540600703587

To link to this article: http://dx.doi.org/10.1080/00207540600703587

曲 Published online: 09 Mar 2007.

Submit your article to this journal $\pi$

Џll Article views: 205

Q View related articles $\asymp$

4 Citing articles: 15 View citing articles $\asymp$ 


\title{
Machining conditions-based preventive maintenance
}

\author{
M. SELIM AKTURK* and SINAN GUREL \\ Department of Industrial Engineering, Bilkent University, 06800 Bilkent, \\ Ankara, Turkey
}

(Revision received February 2006)

\begin{abstract}
In this study we propose an operating conditions-based preventive maintenance (PM) approach for computer numerical control (CNC) turning machines. A CNC machine wears according to how much it is used and the conditions under which it is used. Higher power or production rates result in more wear and higher failure rates. This relationship between the operating conditions and maintenance requirements is usually overlooked in the literature. On CNC turning machines we can control the machining conditions such as cutting speed and feed rate, which in turn affect the PM requirements of the CNC machine. We provide a new model to link the PM decisions to the machining conditions selection decisions, so that these two decision-making problems can be solved together by considering their impact on each other. We establish that our proposed geometric programming model captures the related cost terms along with the technological restrictions of $\mathrm{CNC}$ machines. The proposed preventive maintenance index function can be used to provide an intelligent $\mathrm{CNC}$ machine degradation assessment.
\end{abstract}

Keywords: Preventive maintenance; Condition-based maintenance; CNC machines; Machining conditions selection; Geometric programming

\section{Introduction}

Preventive maintenance (PM) is a set of tasks that are designed to preserve and increase equipment reliability. An ideal PM program for a piece of equipment provides a significant increase in its production capability by preventing failures. In PM applications, when to overhaul a machine is a critical decision. We can observe, even in our everyday life, that if we run a machine under harsh conditions we need to inspect and repair it more often. This relationship between the operating conditions of a machine and its preventive maintenance requirements is usually overlooked in the literature on the process planning and scheduling problems of computer numerical control (CNC) machines. In this paper we propose a new method to use this relationship to develop a condition-based maintenance policy for CNC turning machines.

Based on a survey of plant managers and maintenance managers, Swanson (1999) concludes that advanced manufacturing technology requires more extensive use of

*Corresponding author. Email: akturk@bilkent.edu.tr 
preventive maintenance and computerized maintenance management systems (CMMS). On CNC cutting machines, machining conditions (such as cutting speed and feed rate) are controllable variables. These machines require careful scheduling of operations, tool changes and maintenance to achieve maximum utilization. Since the machining conditions for an operation determine the processing time and the cutting power applied on the machine, they influence the deterioration and its failure risk. Therefore, by applying a machining conditions-based PM to these machines, we can improve their reliability and productivity.

Further motivation for this study is to provide a PM decision tool for a CMMS, namely MAXIMO, which is one of the most popular pieces of CMMS software. Based on the framework provided in this study, an add-on module can be incorporated into CMMS that determines the machining conditions of the manufacturing operations and simultaneously schedules the required PM visits. We know that higher production rates (or shorter processing times) on a CNC machine result in greater deterioration and more frequent PM visits in general. Advances in cutting tool materials and design will increase the cutting speeds at which machining is carried out and consequently reduce the machining time. For example, the effectiveness of ceramic cutting tools is only realized at high cutting speeds. In this study, our aim is to demonstrate the relationship between the PM needs and the operating conditions using a new PM index for a CNC turning machine. This proposed index is calculated for each manufacturing operation that will be processed on the CNC machine and it indicates the PM need of the machine caused by this particular manufacturing operation. The sum of the PM indices of the operations completed since the last PM visit is an indicator for the current PM need of the machine. Based on this, we propose a condition parameter to be monitored to schedule $\mathrm{PM}$ visits on the $\mathrm{CNC}$ machine.

In the literature there are various studies that focus on implementing effective condition-based maintenance approaches. Nolden (1987) discusses predictive maintenance programs that use sophisticated devices (vibration monitoring, wear particle analysers, etc.) to predict failures and problems while the machine is running. Jardine (2002) reviews strategies for implementing smart condition monitoring decisions and focuses on identifying the key risk factors among the signals that are obtained during condition monitoring. Wang (2000) reports a model to determine the optimal critical level and condition reading interval in condition-based maintenance for a criterion of interest. Banjevic et al. (2001) present a control-limit policy and software for condition-based maintenance optimization. Mitchell (1999) surveys the history of condition monitoring and condition-based maintenance. The main question with respect to condition-based maintenance approaches is: how we can define and measure a selected parameter that will help us to understand equipment prognostics? Iakovou et al. (1999) report a throughput-dependent periodic maintenance policy for a general production unit using a Markov decision model. It was based on the idea that higher throughput rates result in greater deterioration of a production system. In the literature there are also studies that integrate PM decisions with various production control decisions. Cassady and Kutanoglu (2003) consider preventive maintenance planning and production scheduling decisions simultaneously so that the total weighted tardiness of jobs is minimized. Kelly et al. (1997) compare the performance of various maintenance policies in conjunction with two-stage scheduling rules in a cellular manufacturing environment. 
In the existing PM approaches, an important parameter is the fixed and known mean time to failure (MTTF), which comes from a certain probability distribution function. This makes sense if we always run the CNC machine at the same cutting speed and feed rate for every manufacturing operation regardless of the work material, surface finish requirements, etc. But, in reality, we can easily change the cutting parameters (i.e. increase or decrease the cutting speed and/or feed rate) to optimize some performance measure. In this study we propose a condition-based maintenance approach. Our main aim is to develop a condition parameter that can be used to determine the PM need of a CNC machine as a function of the machining conditions that we choose for each manufacturing operation.

Mathematical models and solution methods for various machining conditions selection problems for the turning operation are covered by Hitomi (1996). Machining conditions (such as cutting speed and feed rate) identify the cost and production rate for an operation. Malakooti and Deviprasad (1989) formulate the machining conditions selection problem as a multi-objective decision-making problem. Three conflicting objectives of minimizing total cost, production time and surface roughness are considered simultaneously and a heuristic approach is discussed. Gopalakrishnan and Al-Khayyal (1991) provide an analytical tool for the selection of machining conditions in the turning process to minimize total cost of machining and tooling. In these approaches, Taylor's tool life formula is used to represent the relationship between the cutting speed and tool life (Groover 2002).

Recent studies of machining conditions selection problems take into consideration the stochastic nature of the tool life, and relate it to the tool replacement decisions. It is widely agreed that considering the stochastic nature of the tool life gives more accurate results. This idea is supported by the study of Fenton and Joseph (1993), who perform a simulation study to compare deterministic and stochastic approaches. Iakovou et al. (1996) propose models to determine the optimal cutting speed selection and tool replacement policy in machining economics for certain tool life distributions. Kaspi and Shabtay (2003) study the machining economics problem together with the tool replacement strategies for a multi-stage transfer machine. As discussed in Gray et al. (1993), the preventive maintenance and tool replacement decisions are solved at the different levels of the decision-making hierarchy such that the preventive maintenance decisions are handled at a higher level than the tool replacement decisions. In general, there could be several tool replacements (either due to tool wear or change in a part mix) in between two successive preventive maintenance activities. Our proposed model for the preventive maintenance decisions in this study assumes the failure replacement strategy to be applied for the tool replacement decisions.

The studies that consider the stochastic tool life generally assume that there is a single tool type that is used to machine a set of identical parts. Consequently, all the parts use the same operating conditions, such as a constant cutting speed and feed rate on a given machine. This might be an acceptable assumption for tool replacement decisions that are solved in a shorter planning horizon (probably in terms of several hours), but may not be realistic for the preventive maintenance decisions that are usually solved for a longer planning horizon that includes several weeks. Moreover, we usually deal with a low-volume and high-variety production in a flexible manufacturing environment where each job may have a different length, diameter, material type, depth of cut or surface finish requirements. 
Furthermore, each job may require a different type of cutting tool. Therefore, we have to make cutting speed and feed rate decisions for each manufacturing operation independently by solving operation-specific machining economics problems having different objective function parameters and constraints. To the best of our knowledge, for such a low-volume high-variety production, our study is the first attempt to consider a condition-based maintenance policy in a machining economics model.

In the next section we first characterize the mathematical relationship between the PM cost and production rate for a CNC turning machine. We introduce a new PM index for a manufacturing operation and demonstrate how this index can be used in a condition-based maintenance policy. In section 3 we discuss the machining conditions selection problem of minimizing manufacturing and PM costs simultaneously for a single machining operation, and derive an optimality condition for the problem. In section 4 we provide an algorithm for solving the joint problem. In section 5 a numerical example is presented. Finally, in section 6 we investigate the impact of using different objective functions in the machining conditions selection problem on the scheduling performance of a $\mathrm{CNC}$ machine.

\section{Proposed PM index}

The notation used throughout the paper is as follows.

Parameters

$A, B, k, T$ coefficients and exponents for the proposed PM index function

$\alpha_{j}, \beta_{j}, \gamma_{j}$ speed, feed, depth of cut exponents for tool $j$

$K_{j} \quad$ Taylor's tool life constant for tool $j$

$C_{\mathrm{m}}, b, c, e$ specific coefficients and exponents of the machine power constraint

$C_{\mathrm{s}}, g, h, l$ specific coefficients and exponents of the surface roughness constraint

$C_{\mathrm{o}}$ operating cost of the CNC machine $(\$ / \mathrm{min})$

$C_{\mathrm{t}_{j}} \quad$ cost of tool $j(\$ /$ tool)

$D_{i}$ diameter of the generated surface for operation $i$ (in.)

$d_{i}$ depth of cut for operation $i$ (in.)

$H$ maximum available machine power (hp)

$L_{i}$ length of the generated surface for operation $i$ (in.)

$S_{i}$ maximum allowable surface roughness for operation $i$ ( $\mu$ in.)

$C_{\mathrm{PM}} \quad$ cost of a $\mathrm{PM}$ visit (\$/visit)

$\tau_{\mathrm{pm}}$ duration of a PM visit (min)

$t_{\mathrm{r}} \quad$ tool replacement time $(\mathrm{min})$

Decision variables

$v_{i j} \quad$ cutting speed for operation $i$ using tool $j$ (fpm)

$f_{i j} \quad$ feed rate for operation $i$ using tool $j$ (ipr)

Dependent variables

$t_{m_{i j}}$ processing time of operation $i$ using tool $j$ (min)

$r_{i}$ production rate of the machine for operation $i$ (part $/ \mathrm{min}$ )

$Z_{i j} \quad$ expected tool life of tool $j$ for operation $i$ (min)

$U_{i j} \quad$ expected usage rate of tool $j$ for operation $i$

$P_{i j} \quad$ PM index for operation $i$ when tool $j$ is used 
On a CNC turning machine there is a set of jobs to be processed and each job corresponds to a metal cutting operation that can be performed by a different cutting tool. The processing time of an operation can be decreased by increasing the cutting speed and/or feed rate:

$$
t_{m_{i j}}=\frac{\pi D_{i} L_{i}}{12 v_{i j} f_{i j}} .
$$

During operation there is a continual wearing action that occurs on the cutting tool. This wear leads to failure. We assume a failure replacement strategy for the tool replacement decisions, so that a tool is replaced only when it fails, i.e. a predetermined level of tool wear is reached. Shabtay and Kaspi (2002) discuss the failure replacement strategy for tool replacements on cutting machines. The time between two successive tool replacements, or the expected tool life for a given operation, can be approximated by Taylor's tool life formula. For further discussion on Taylor's tool life formula, we refer to Groover (2002). Expected tool usage for a cutting operation can be calculated using the above processing time equation and the expected tool life calculated using Taylor's tool life formula:

$$
U_{i j}=\frac{t_{m_{i j}}}{Z_{i j}}=\frac{\left(\pi D_{i} L_{i}\right) /\left(12 v_{i j} f_{i j}\right)}{K_{j} /\left(v_{i j}^{\alpha_{j}} f_{i j}^{\beta_{j}} d_{i}^{\dot{\gamma}_{j}}\right)} .
$$

In order to increase the production rate on a $\mathrm{CNC}$ turning machine at a given time period we need to run the machine at higher power levels due to the increased cutting speed and feed rate. This means a greater force and a higher temperature acting on the machine. Therefore, more wear takes place on the machine and the risk of sudden failure increases. The machine needs to be overhauled more often, which results in a higher PM cost. At higher production rates, the machine is more sensitive and unstable, so that increasing the production rate further causes greater deterioration and higher failure risks. At higher production rates, the marginal increase in the PM cost is higher.

Moreover, if a machine is idle, we have to service it so as to keep it ready to operate whenever necessary and to prevent wear over time. An idle machine must be cleaned and inspected periodically, so that it is not left to wear out on the shop floor. Therefore, for a given time period we have a fixed minimum PM cost even when the production rate is zero.

In this study, we assume that, for a given operating time period $T$ for a $\mathrm{CNC}$ turning machine, the form of the PM cost function is

$$
\mathrm{PM} \operatorname{cost}=A+B r^{k},
$$

where $r$ is the production rate of the machine (parts/min) and $A$ is the PM cost of the machine when it is idle for an operating time period of T. $B$ and $k$ are dependent on $T$ and on the cost of a single PM visit, $C_{\mathrm{PM}}$. These parameters are specific to the considered machine's condition. An older machine would have higher $B$ and $k$ since it would require more frequent PM visits. They are also affected by the hardness (or strength) of the required cutting tool and work material combination. Similar to Taylor's tool life formula, we can determine the constant parameter $B$ and exponent $k$ empirically based on past shop floor data. Furthermore, this function is increasing and convex, so we have $B>0$ and $k \geq 1$. We can express the production 
rate of the machine for each operation as $r=1 / t_{\mathrm{m}}$. Then, our cost function can be written as

$$
\mathrm{PM} \text { cost }=A+B / t_{\mathrm{m}}^{k}
$$

As we know the total PM cost for an operating period of length $T$ and processing time $t_{\mathrm{m}}$ for the operations in this period, under the failure replacement strategy for tool replacement decisions we can find the expected PM cost per operation by dividing the total PM cost for the period by the expected number of operations completed in the period, which is $n=T /\left(t_{\mathrm{m}}+t_{\mathrm{r}} \cdot U\right)$. Then we can calculate the expected PM cost per operation:

$$
\text { PM cost per operation }=\frac{1}{T}\left(A \cdot t_{\mathrm{m}}+A \cdot t_{\mathrm{r}} \cdot U+\frac{B}{t_{\mathrm{m}}^{(k-1)}}+\frac{B \cdot t_{\mathrm{r}} \cdot U}{t_{\mathrm{m}}^{k}}\right) .
$$

We now derive a PM cost function of an operation from the PM cost function for a machine by assuming that the machine runs at a constant production rate for operating time $T$. However, in practice we do not operate all of the operations at the same production rate. Each operation's machining conditions are decided individually based upon the work material and design parameters. While performing these operations with different processing times, we need a measure to estimate each operation's contribution to the PM need of the CNC machine. For a further discussion on the set of tasks to be performed in a typical PM visit of a CNC machine, we refer to Higgins et al. (1995). We assume that the PM visit cost $\left(C_{\mathrm{PM}}\right)$ is constant for all visits. We determine the contribution of an operation to the PM need of a machine using the ratio of the PM cost of an operation to $C_{\mathrm{PM}}$. This is a value between 0 and 1 , and gives a measure of what proportion of a PM visit cost is due to the considered manufacturing operation. The general expression for the proposed PM index of each operation, $P_{i j}$, described above is

$$
\mathrm{PM} \text { index }=\frac{1}{T \cdot C_{\mathrm{PM}}}\left(A \cdot t_{\mathrm{m}}+A \cdot t_{\mathrm{r}} \cdot U+\frac{B}{t_{\mathrm{m}}^{(k-1)}}+\frac{B \cdot t_{\mathrm{r}} \cdot U}{t_{\mathrm{m}}^{k}}\right),
$$

which, in terms of $v_{i j}$ and $f_{i j}$, can be expressed as

$$
P_{i j}=P_{1} v_{i j}^{-1} f_{i j}^{-1}+P_{2} v_{i j}^{\alpha_{j}-1} f_{i j}^{\beta_{j}-1}+P_{3} v_{i j}^{k-1} f_{i j}^{k-1}+P_{4} v_{i j}^{\alpha_{j}+k-1} f_{i j}^{\beta_{j}+k-1},
$$

where

$$
\begin{aligned}
& P_{1}=\frac{A \pi D_{i} L_{i}}{12 T C_{\mathrm{PM}}}, \quad P_{2}=\frac{A t_{\mathrm{r}} \pi D_{i} L_{i} d^{\gamma_{j}}}{12 K_{j} T C_{\mathrm{PM}}}, \\
& P_{3}=\frac{B}{T C_{\mathrm{PM}}}\left[\frac{12}{\pi D_{i} L_{i}}\right]^{(k-1)}, \quad P_{4}=\frac{B t_{\mathrm{r}} d^{\gamma_{j}}}{K_{j} T C_{\mathrm{PM}}}\left[\frac{12}{\pi D_{i} L_{i}}\right]^{(k-1)} .
\end{aligned}
$$

For a set of operations that are processed sequentially, we can easily calculate the PM index for each operation and use these indices to schedule the required PM visits. When the sum of the PM indices of the operations completed since the previous PM visit reaches 1, we schedule a PM visit. Let us demonstrate these calculations on a small example. Suppose that we have a PM index function for the jobs on a CNC machine where $A=5, B=1800, k=2.5, C_{\mathrm{PM}}=5, T=750$ and $t_{\mathrm{r}}=1$. 
Then the PM index function is

$$
\mathrm{PM} \text { index }=\frac{1}{3750}\left(5 \cdot t_{\mathrm{m}}+5 \cdot U+\frac{1800}{t_{\mathrm{m}}^{1.5}}+\frac{1800 \cdot U}{t_{\mathrm{m}}^{2.5}}\right) .
$$

If we set $(v, f)$ of an operation to achieve $t_{\mathrm{m}}=2$ and $U=0.01$, then the PM index is equal to 0.173 . Then we have to make a PM visit after $(1 / 0.173=5.78)$ (or approximately after five jobs). If we operate at $t_{\mathrm{m}}=4$ and $U=0.005$, then the PM index is 0.065 and we have to maintain the machine after 15 jobs (since $1 / 0.065=15.38)$.

We define the PM cost of an operation as a function of the machining conditions $(v, f)$. In the next section, we will discuss how we can calculate the machining conditions for each manufacturing operation.

\section{Single machining operation problem (SMOP)}

In the preceding sections we have derived mathematical expressions for the PM cost and the PM index of an operation. The main idea behind the single machining operation problem (SMOP) is to determine the optimum machining conditions (i.e. cutting speed and feed rate) for each manufacturing operation to optimize some performance measure subject to the constraints that the selected machining conditions do not exceed the available machine power and cutting tool life and satisfy the necessary quality requirements for each operation. To the best of our knowledge, maintenance-related cost parameters are not considered in the SMOP formulations in the existing literature. Gopalakrishnan and Al-Khayyal (1991) and Akturk and Avci (1996) are two examples that study the SMOP with only machining and tooling cost terms. We provide a new mathematical model for the SMOP that includes a PM cost term in the objective function and prove an optimality condition for the new problem. Moreover, we present a new solution procedure to solve this problem in section 4 .

On a CNC turning machine, for a given operation $i$ using tool $j$, we have three cost terms, the machining cost, the tooling cost and the PM cost. The machining cost is a function of the processing time. The tooling cost is a function of the expected tool usage and the PM cost is a function of the PM index. We can control the processing time, the expected tool usage and the PM index for each operation by controlling the cutting speed and the feed rate of the cutting operation on a CNC turning machine.

The geometric programming (GP) model for the problem is as follows:

$$
\begin{aligned}
(\mathrm{GP}) \operatorname{minimize} M_{i j}= & C_{\mathrm{o}} \cdot t_{m_{i j}}+C_{\mathrm{t}} \cdot U_{i j}+C_{\mathrm{PM}} \cdot P_{i j} \\
= & C_{1} v_{i j}^{-1} f_{i j}^{-1}+C_{2} v_{i j}^{\left(\alpha_{j}-1\right)} f_{i j}^{\left(\beta_{j}-1\right)}+C_{3} v_{i j}^{(k-1)} f_{i j}^{(k-1)} \\
& +C_{4} v_{i j}^{(\alpha+k-1)} f_{i j}^{(\beta+k-1)},
\end{aligned}
$$


subject to

$$
\begin{aligned}
C_{\mathrm{t}}^{\prime} v_{i j}^{\alpha_{j}-1} f_{i j}^{\beta_{j}-1} & \leq 1 \quad \text { (tool life constraint) } \\
C_{\mathrm{m}}^{\prime} v_{i j}^{b} f_{i j}^{c} & \leq 1 \quad \text { (machine power constraint) } \\
C_{\mathrm{s}}^{\prime} v_{i j}^{g} f_{i j}^{h} & \leq 1 \quad \text { (surface roughness constraint) } \\
v_{i j}, f_{i j} & >0,
\end{aligned}
$$

where

$$
\begin{aligned}
& C_{1}=\frac{\pi D_{i} L_{i} C_{\mathrm{o}}}{12}+C_{\mathrm{PM}} P_{1}, \quad C_{2}=\frac{\pi D_{i} L_{i} d_{i}^{\gamma_{j}} C_{\mathrm{t}_{j}}}{12 K_{j}}+C_{\mathrm{PM}} P_{2}, \quad C_{3}=C_{\mathrm{PM}} P_{3}, \\
& C_{4}=C_{\mathrm{PM}} P_{4}, \quad C_{\mathrm{t}}^{\prime}=\frac{\pi D_{i} L_{i} d_{i}^{\gamma_{j}}}{12 K_{j}}, \quad C_{\mathrm{m}}^{\prime}=\frac{C_{\mathrm{m}} d_{i}^{e}}{H} \text { and } C_{\mathrm{s}}^{\prime}=\frac{C_{\mathrm{s}} d_{i}^{l}}{S_{i}} .
\end{aligned}
$$

On a CNC turning machine, for each operation, we can set the cutting speed and the feed rate within some constraints. These constraints are due to the technical limitations of the machine and the tool and the design requirements for the part. The first constraint is the tool life constraint, which sets an upper bound for the tool usage, $U_{i j}$, that will occur by the cutting operation. As $v_{i j}$ and $f_{i j}$ increase, tool usage increases. The second constraint is the machine power constraint due to the maximum available horse power of the CNC machine. As $v_{i j}$ and $f_{i j}$ increase, more horse power is required to perform the metal cutting operation. The last constraint is the surface roughness constraint due to the part's design requirement. It sets the maximum allowable surface roughness for each operation. As $v_{i j}$ increases, surface roughness decreases, and as $f_{i j}$ increases, surface roughness increases. These constraints limit the applicable $v_{i j}$ and $f_{i j}$ values for each operation.

In the literature, only the machining and the tooling costs are considered and a corresponding GP model is provided. Here, we additionally include the PM cost in the objective function. Since the PM cost term is a function of $\left(v_{i j}, f_{i j}\right)$, the mathematical model for the new problem is still a GP model. Even so, this new problem is more difficult to solve due to the additional PM cost terms in its objective function. Figure 1 shows an example of the feasible region for SMOP. The region under the constraints is the feasible region where we can select the cutting speed and the feed rate.

Given the above GP model, we will derive conclusions concerning the optimal solution to the problem by using duality and complementary slackness properties. The dual of a geometric program has linear constraints. This, together with complementary slackness properties, will help us to solve this problem. Denoting the dual variables by $Y_{1}, Y_{2}, \ldots, Y_{7}$ the dual formulation for the SMOP problem can be written as

$$
\operatorname{maximize}\left(\frac{C_{1}}{Y_{1}}\right)^{Y_{1}} \cdot\left(\frac{C_{2}}{Y_{2}}\right)^{Y_{2}} \cdot\left(\frac{C_{3}}{Y_{3}}\right)^{Y_{3}} \cdot\left(\frac{C_{4}}{Y_{4}}\right)^{Y_{4}} \cdot\left(C_{\mathrm{t}}^{\prime}\right)^{Y_{5}} \cdot\left(C_{\mathrm{m}}^{\prime}\right)^{Y_{6}} \cdot\left(C_{\mathrm{s}}^{\prime}\right)^{Y_{7}},
$$


(a) Feed rate

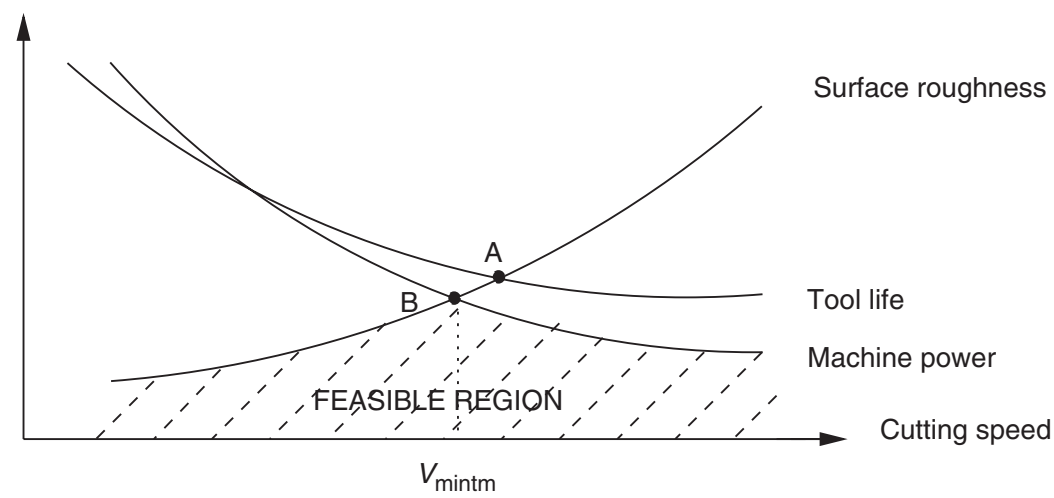

(b) Feed rate

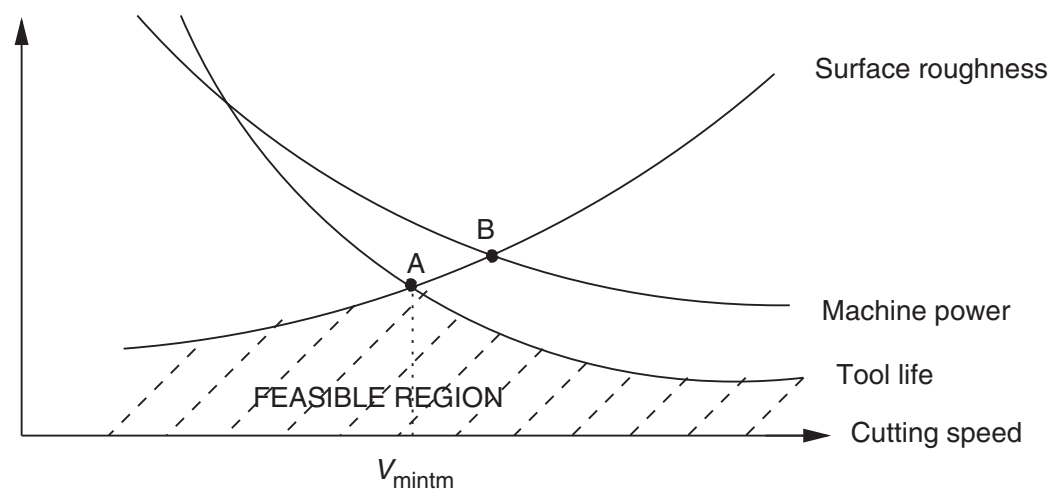

Figure 1. A typical feasible region for SMOP.

subject to

$$
\begin{aligned}
& Y_{1}+Y_{2}+Y_{3}+Y_{4}=1, \\
& -Y_{1}+\left(\alpha_{j}-1\right) \cdot Y_{2}+(k-1) \cdot Y_{3}+(\alpha+k-1) \cdot Y_{4}+\left(\alpha_{j}-1\right) \\
& \quad \cdot Y_{5}+b \cdot Y_{6}+g \cdot Y_{7}=0, \\
& -Y_{1}+\left(\beta_{j}-1\right) \cdot Y_{2}+(k-1) \cdot Y_{3}+(\beta+k-1) \cdot Y_{4}+\left(\beta_{j}-1\right) \\
& \quad \cdot Y_{5}+c \cdot Y_{6}+h \cdot Y_{7}=0, \\
& Y_{1}, Y_{2}, Y_{3}, Y_{4}, Y_{5}, Y_{6}, Y_{7} \geq 0 .
\end{aligned}
$$

The first four dual variables, $Y_{1}, Y_{2}, Y_{3}$ and $Y_{4}$, correspond to each of the primal objective function terms. Therefore, their summation must be equal to 1 , also known as the normality constraint, as stated in the first dual constraint. The other dual variables, $Y_{5}, Y_{6}$ and $Y_{7}$, correspond to the primal problem constraints. Furthermore, there is a dual constraint for each primal variable, $v_{i j}$ and $f_{i j}$, known as orthogonality constraints. Each of the constraints of the primal problem can be either loose or tight at optimality. The following theorem will be very helpful in reducing the search space. 


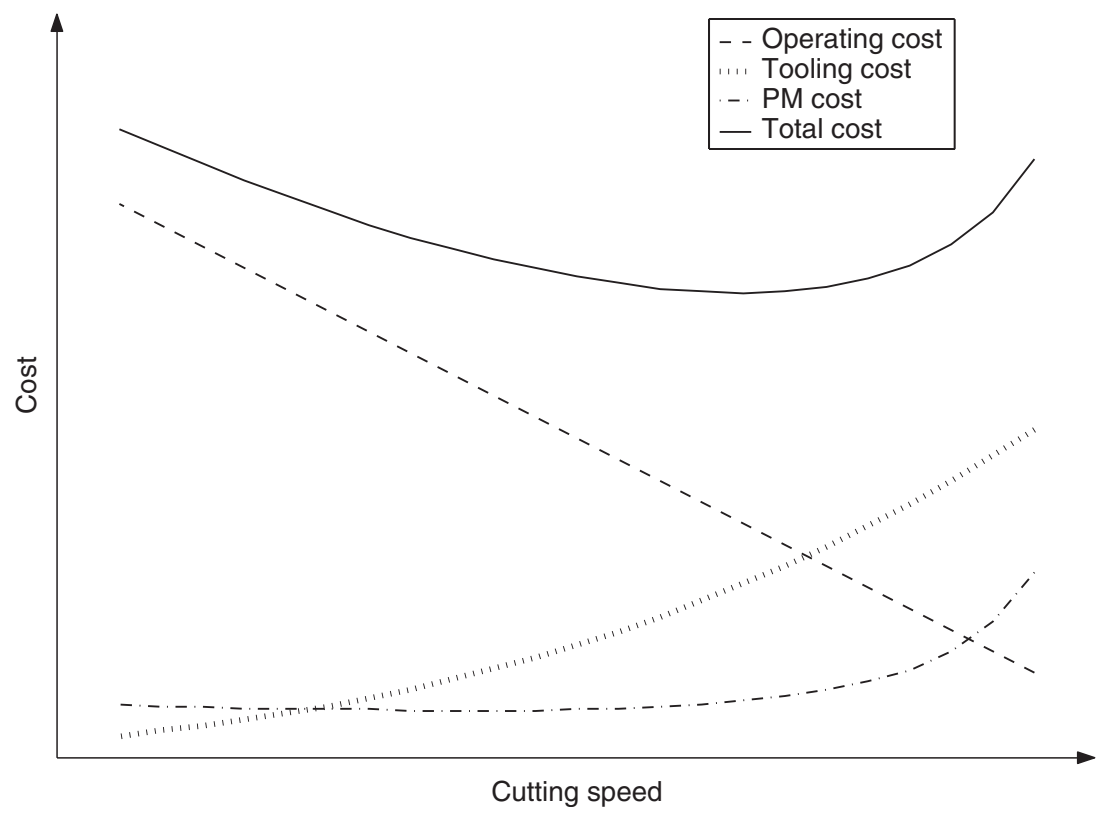

Figure 2. Different cost terms for SMOP versus cutting speed.

Theorem 3.1: The surface roughness constraint must be tight at the optimal solution.

Proof: Suppose that, at an optimal solution, the surface roughness constraint is loose. Then, the corresponding dual variable for the surface roughness constraint, $Y_{7}$, is zero due to the complementary slackness conditions. When $Y_{7}=0$, dual constraints (10) and (11) and the dual non-negativity constraints cannot be satisfied at the same time since $\alpha_{j}>\beta_{j}>1$ and $b>c>0$ due to Gorczyca (1987) (this means that an increasing cutting speed or feed rate always requires more machine power and tool usage). Moreover, machine power and tool life are more sensitive to changes in the cutting speed than the feed rate, yielding $b>c>0$ and $\alpha_{j}>\beta_{j}>1$. Consequently, the dual problem is infeasible when $Y_{7}=0$. This proves that, at optimality, the surface roughness constraint must be tight.

Having shown that the surface roughness constraint is tight at the optimal solution for the SMOP, the next step is to see how the objective function and its terms behave with respect to the surface roughness constraint. Figure 2 shows the machining cost, tooling cost, PM cost and total cost as a function of the cutting speed. We know that the machining cost, tooling cost and PM cost functions are convex. Then, our objective function, being the sum of convex functions, is also convex.

In figure 1, suppose that we are moving along the surface roughness constraint in the direction where both the cutting speed $(v)$ and the feed rate $(f)$ increase. If $v$ and $f$ increase, then $t_{\mathrm{m}}$ decreases, and therefore the machining cost decreases. The tooling cost increases because $U_{i j}$ increases, since $\alpha_{j}, \beta_{j}>1$. The PM cost first decreases, reaches a minimum level and then increases with increasing $v$. 
This is because, even if we keep the machine idle, we have to pay for its PM in order to keep it ready to operate. Also, this form shows that, at cutting speeds, equivalently for lower processing times, the marginal increase in the PM cost is higher. Since we added the PM cost term to the objective function of the SMOP, the number of terms in the geometric program increased by 2 . This increased the degree of difficulty of the geometric programming formulation to four. Therefore, the problem is harder than that without a PM cost term in the objective function. For this new problem, we have provided a new solution method as discussed below.

\section{Solution procedure}

As a result of theorem 3.1, we know that the surface roughness constraint is always tight at optimality. Using this, we obtain a one-dimensional cost function for the surface roughness constraint since we can derive an analytical expression for the feed rate as a function of the cutting speed. Let $f_{i j}=\left(1 /\left(C_{\mathrm{s}}^{\prime} \cdot v_{i j}^{g}\right)\right)^{1 / h}$ due to theorem 3.1 , then the objective function becomes

$$
\begin{aligned}
M_{i j}(v)= & C_{1}\left(C_{\mathrm{s}}^{\prime}\right)^{(1 / h)} v_{i j}^{(g-h) / h}+C_{2}\left(C_{\mathrm{s}}^{\prime}\right)^{-\left(\beta_{j}-1\right) / h} v_{i j}^{\left[h\left(\alpha_{j}-1\right)-g\left(\beta_{j}-1\right)\right] / h} \\
& +C_{3}\left(C_{\mathrm{s}}^{\prime}\right)^{-(k-1) / h} v_{i j}^{[(h-g)(k-1)] / h} \\
& +C_{4}\left(C_{\mathrm{s}}^{\prime}\right)^{-(\beta+k-1) / h} v_{i j}^{[h(\alpha+k-1)-g(\beta+k-1)] / h}
\end{aligned}
$$

Since the objective is a convex function and due to the fact that we cannot find an explicit closed-form expression for the optimal cutting speed, we can use any one-dimensional search algorithm, such as the Golden Section Algorithm (GSA) or bisection method, to determine the optimal solution. A more detailed discussion on the GSA and bisection method can be found in Bazaraa et al. (1993).

To apply a line search algorithm, we first find an upper bound and a lower bound for the optimal cutting speed, $v_{\text {opt }}$. The objective function is the sum of two convex functions: the PM cost and the machining plus tooling cost. Let $v_{m+t}$ be the cutting speed that minimizes the machining plus tooling cost. We could have an upper bound on $v_{\text {opt }}$, which is $v_{\text {mintm }} . v_{\text {mintm }}$ is the cutting speed that corresponds to the minimum processing time achievable for each operation. Therefore, there are two possibilities: either we find a search interval including $v_{\text {opt }}$ or we conclude that $v_{\text {opt }}=v_{\text {mintm }}$. Since we use the same algorithm for each operation, we will omit the indices $(i, j)$ from the algorithmic description for the sake of clarity and simplicity.

The proposed algorithm for solving the SMOP is as follows.

- Step 1. Find the $(v, f)$ pair where both the surface roughness and the machine power constraints are tight. this corresponds to point $\mathrm{B}$ in figure 1(a). Using the equalities for both constraints, the corresponding expressions for $v$ and $f$ are

$$
v_{\mathrm{B}}=\left(\frac{1}{C_{\mathrm{s}}^{\prime} f^{h}}\right)^{1 / g} \text { and } f_{\mathrm{B}}=\left[\left(C_{\mathrm{m}}^{\prime}\right)^{g}\left(C_{\mathrm{s}}^{\prime}\right)^{-b}\right]^{1 /(b h-g c)} .
$$



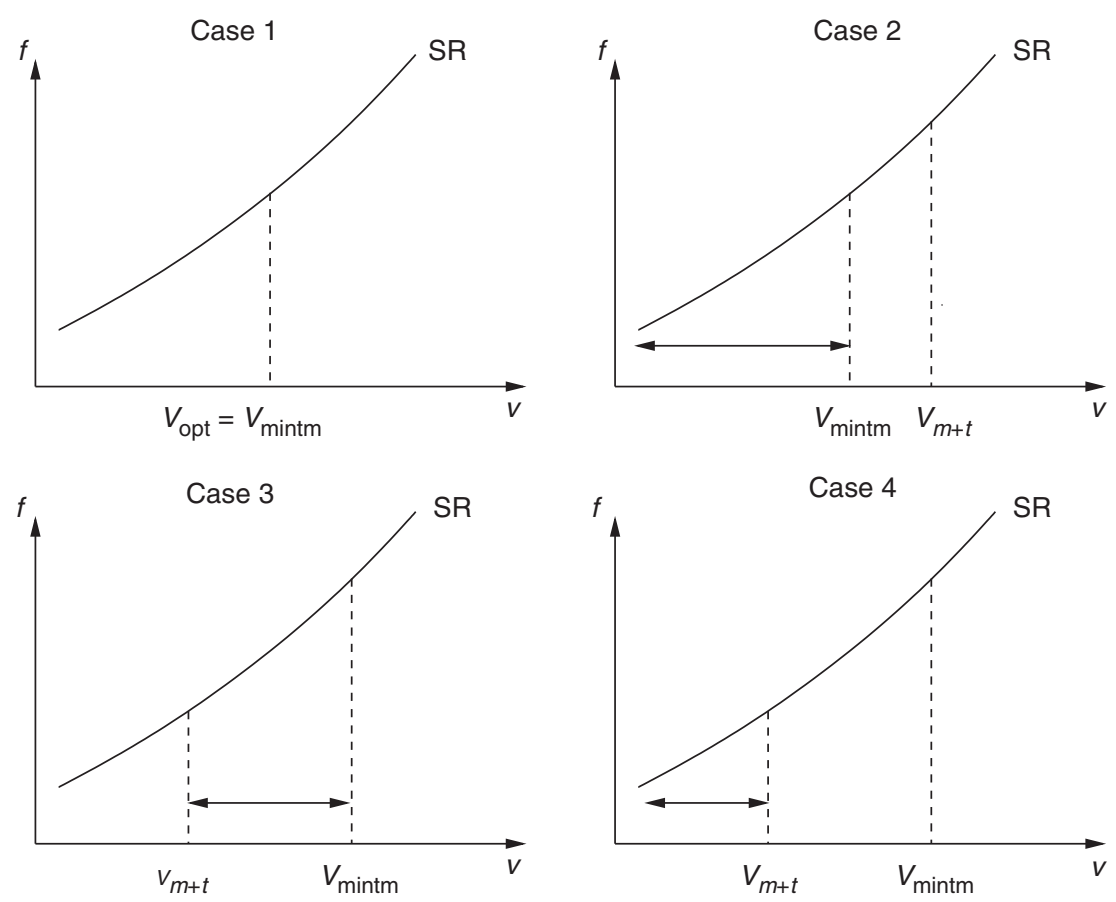

Figure 3. Possible search intervals to perform GSA.

- Step 2. Find the $(v, f)$ pair where both the surface roughness and the tool life constraints are tight. This corresponds to point A in figure 1(b). Therefore,

$$
v_{\mathrm{A}}=\left(\frac{1}{C_{\mathrm{s}}^{\prime} f^{h}}\right)^{1 / g} \text { and } f_{\mathrm{A}}=\left[\left(C_{\mathrm{s}}^{\prime}\right)^{\alpha-1}\left(C_{\mathrm{t}}^{\prime}\right)^{-g}\right]^{1 /[g(\beta-1)-h(\alpha-1)]} .
$$

- Step 3. Determine the cutting speed for the minimum processing time, $v_{\operatorname{mintm}}=\min \left\{v_{\mathrm{A}}, v_{\mathrm{B}}\right\}$.

- Step 4. If $M^{\prime}\left(v_{\operatorname{mintm}}\right) \leq 0$, then $v_{\mathrm{opt}}=v_{\operatorname{mintm}}$ (Case 1 in figure 3), and go to Step 8. Else, go to Step 5.

- Step 5. Relax the tool life and machine power constraints and find the $(v, f)$ pair that minimizes the machining plus tooling cost on the surface roughness constraint. Since the surface roughness constraint is tight we can write $f$ in terms of $v$ as in Step 4. Due to Akturk and Avci (1996), by using the expression for $f$, we can derive the objective function on the surface roughness constraint. By taking the derivative of this function for $v$ and setting it to zero, the optimal $v$ for this case is

$$
v_{m+t}=\left[\frac{C_{1}}{C_{2}}\left(C_{\mathrm{s}}^{\prime}\right)^{\left(\beta_{j} / h\right)} \frac{h-g}{h\left(\alpha_{j}-1\right)-g\left(\beta_{j}-1\right)}\right]^{h /\left(h \alpha_{j}-g \beta_{j}\right)} .
$$

- Step 6. Find the search interval for the cutting speed $\left(v_{\mathrm{u}}\right.$, upper limit; $v_{\mathrm{l}}$, lower limit) to apply the line search algorithm:

If $v_{\text {mintm }}<v_{m+t}$, then $v_{1}=0$ and $v_{\mathrm{u}}=v_{\text {mintm }}$ (Case 2 in figure 3), 
else if $M^{\prime}\left(v_{m+t}\right)<0$, then $v_{1}=v_{m+t}$ and $v_{\mathrm{u}}=v_{\text {mintm }}$ (Case 3 in figure 3), else if $M^{\prime}\left(v_{m+t}\right)<M^{\prime}\left(v_{\text {mintm }}\right)$, then $v_{1}=0$ and $v_{\mathrm{u}}=v_{m+t}$ (Case 4 in figure 3).

- Step 7. The optimal solution to this problem is obtained by applying any one-dimensional search procedure over all possible values of $v$. For example, according to the bisection method (Bazaraa et al. 1993), the optimal value of $v$ can be found in $\log \left[\left(v_{u}-v_{1}\right) / \epsilon\right]$ iterations within an accuracy of $\epsilon$.

- Step 8. Having found the optimal cutting speed, determine the corresponding feed rate and $t_{\mathrm{m}}$ on the surface roughness constraint.

In the first three steps of the proposed algorithm, we find $v_{\text {mintm. }}$. This is the cutting speed that gives the minimum processing time on the surface roughness constraint (the lower bound on the processing time, but the upper bound on the cutting speed for each operation). To find $v_{\text {mintm }}$, we should consider two different cases. One is where $v_{\text {mintm }}$ is at the point where both the surface roughness and the machine power constraints are tight (Step 1). The other case is where $v_{\text {mintm }}$ is at the point where both the surface roughness and the tool life constraints are tight (Step 2). Figures 1(a) and (b) present these two cases, respectively. In Step 3, we determine $v_{\text {mintm }}$.

In Step 4, we check if $v_{\text {mintm }}$ is the optimal cutting speed or not. If so, then we do not need to apply any search because $v_{\text {mintm }}$ is the optimal cutting speed. This step covers Case 1 of figure 3. In Step 5, we find $v_{m+t}$. We find a search interval to perform the line search in Step 6. In Step 6, Cases 2-4 of figure 3 are considered. In Step 7, the line search algorithm is applied to find $v_{\text {opt }}$. Finally, in Step 8, we calculate the optimal $f$ and the corresponding optimal $t_{\mathrm{m}}$ values.

\section{Numerical example}

In this section we provide a numerical example to demonstrate the proposed solution procedure. We have a manufacturing operation $i$ with $D_{i}=8, L_{i}=6, d_{i}=0.08$ and $S_{i}=300$. We use cutting tool $j$ with $\alpha_{j}=3.9, \beta_{j}=1.30, \gamma_{j}=1.1$ and $C_{j}=125321000$ to process this operation. The tool replacement time is $t_{\mathrm{r}}=1$. The technological coefficients of the machine power constraint are $b=0.91, c=0.78, e=0.75$ and $C_{\mathrm{m}}=2.394$. The surface roughness-related coefficients are $g=-1.52, h=1.004$, $l=0.25$ and $C_{\mathrm{s}}=204620000$. We have the PM index function coefficients $A=10$, $B=15, T=2000$ and $k=2.5$. The cost of a PM visit is $C_{\mathrm{PM}}=15$. The operating cost is $C_{\mathrm{o}}=0.1$ and the tool cost is $C_{\mathrm{t}}=6$. We consider a CNC turning machine with a maximum horsepower of $H=15$.

In Step 1 we find $v_{\mathrm{B}}=692$ and $f_{\mathrm{B}}=0.06$, where the surface roughness and machine power constraints are tight. In Step 2 we find $v_{\mathrm{A}}=874$ and $f_{\mathrm{A}}=0.08$, where the surface roughness and tool life constraints are tight. In Step 3 we set $v_{\text {mintm }}=692$. In Step 4 we find that $M^{\prime}\left(v_{\text {mintm }}\right)=0.014$, therefore $v_{\text {mintm }}$ is not the optimal cutting speed and we move to Step 5. In Step 5 we find the cutting speed that minimizes the machining plus tooling cost on the surface roughness constraint: $v_{m+t}=307$. In Step 6 we determine the upper and lower bounds of the interval the line search will be applied on. Since $v_{\text {mintm }}>v_{m+t}$ we find $M^{\prime}\left(v_{m+t}\right)=-0.00007$. We then conclude that $v_{m+t}<v_{\text {opt }}<v_{\text {mintm }}$ and set $v_{1}=307$ and $v_{\mathrm{u}}=692$. 
In Step 7 we implement a line search algorithm to find the optimum cutting speed, which is $v_{\text {opt }}=310$, and then we calculate the optimum feed rate $f_{\text {opt }}=0.02$ and the corresponding $t_{\mathrm{m}}=2.37$. At this point, the total cost of this manufacturing operation is 0.44 , the tool usage is 0.03 and the PM index is 0.0009 . For the continuous production of a single item, the expected time between two tool replacements would be $79 \mathrm{~min}$, whereas the time between two PM visits would be $(2.37 / 0.0009)=$ $2633 \mathrm{~min}$ (or under the assumption of $480 \mathrm{~min} /$ day, 5.5 days).

Let us now demonstrate how these results can be implemented in practice. The machining settings of a CNC machine are very easy to change by a single line in a $\mathrm{G}$ code or in an APT language. The feed rate is the speed of the cutting tool moving along the part profile or from one point to another. It is defined as the distance (in inches or millimeters) that the tool moves in 1 min or in one revolution of the machine tool spindle. In this paper, we measured the feed rate in inches per minute (ipr). For example, the following $\mathrm{G}$ code will be included in the NC program to set the feed rate:

\section{G99 F0.02;}

in the APT language, we have to add the following statement:

\section{FEDRAT/0.02, IPR}

On the other hand, in any CMMS application (such as MAXIMO), we have to keep track of the accumulated PM indices for each CNC machine. As a result, we can automatically generate the PM requests based upon the operating conditions of the CNC machine, thus bringing down the cost of maintenance operations while avoiding unnecessary maintenance (this might happen if we maintain every $\mathrm{CNC}$ machine with a fixed interval between visits as in regular PM activities as opposed to condition-based maintenance). Another benefit could be improved availability of the CNC machines (resulting from reduced or eliminated machine breakdowns). Consequently, the proposed PM index function for each manufacturing operation can be used to provide an intelligent $\mathrm{CNC}$ machine degradation assessment.

\section{Computational results}

In this study, besides the new machining conditions-based PM approach, we have discussed a new machining conditions selection problem for the turning operation. In this section we investigate how the scheduling decisions on a single CNC machine can change with respect to a different performance measure at the SMOP level. In each case, the machining conditions of the jobs are decided by solving the SMOP for a different objective function to demonstrate the sensitivity of the completion times to the selected objective function.

We propose a new mathematical model of the SMOP with the objective function of machining plus tooling plus PM costs. We call this case $M+T+P M$. Akturk and Avci (1996) provided a solution method for the SMOP with the objective function of machining plus tooling costs. We called this case $M+T$. For scheduling related measures, we should consider the minimum processing time case (denoted $P l$ ). Another objective could be minimizing the PM cost or the PM index itself. 


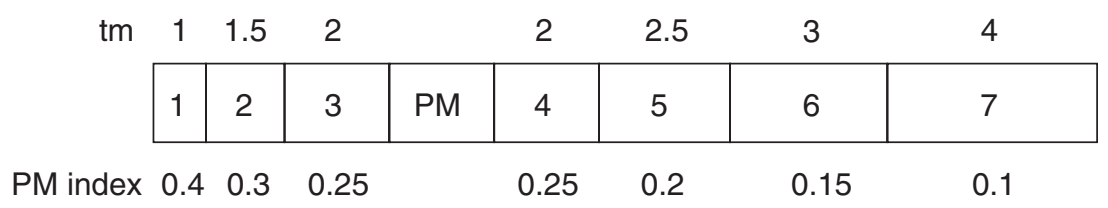

Figure 4. A Schedule with a PM visit.

Solving the SMOP to minimize the PM index given in equation (7) is a difficult problem since it has the same degree of difficulty as in the $M+T+P M$ case. This is because there are four $(v, f)$ terms in the objective function of equation (7) and the solution must satisfy the same set of constraints as in the $M+T+P M$ case. It is easy to show that theorem 3.1 also holds for the problem of minimizing the PM index. Therefore, we designed another algorithm that employs a line search algorithm to solve this problem in our computational study. We called this case $P M$. The last objective function we considered in SMOP was machining plus PM cost. This case also has the same degree of difficulty as the $M+T+P M$ case. Theorem 3.1 also holds for this case, therefore we employed another algorithm to solve it in our computational study. We call this case $M+P M$.

We consider the total completion time measure for a given set of jobs (each job corresponds to a manufacturing operation as discussed earlier). The completion time is a measure of the time that the job stays in the system. It is a measure of the workin-process inventory cost. We use the total completion time as a surrogate measure for the different cases discussed above. For each objective function, we solve the SMOP and determine the optimal processing time and the PM index of each operation, then we order the jobs by the shortest processing time (SPT) rule and schedule PM visits between the jobs when needed. We also consider tool replacements and use the expected tool usages of jobs to schedule tool replacements. In our experiments we assume a deterministic tool replacement process. We insert tool replacement periods in a schedule so that, between two tool replacements, the total expected tool usage of jobs cannot exceed 1 . There could be some CNC machines that allow off-line tool replacements, for which tool replacement times can be negligible. But, in general, tool replacement times are significantly shorter than the PM visit durations. Having formed a schedule for each case, we evaluate the total completion time for each schedule. We observe how each objective function affects the total completion time under the SPT sequence.

Figure 4 shows an example of a schedule of jobs with zero tool replacement times for the sake of simplicity. Each job is assigned a $t_{\mathrm{m}}$ and a corresponding PM index. The jobs are ordered by the SPT rule. The sum of the PM indices of jobs 1 to 3 is 0.95 , so that a PM visit is scheduled after job 3 . The PM visit duration is 2 , so the completion times for the jobs are $1,2.5,4.5,8.5,11,14$ and 18 , respectively. The total completion time of the given schedule is 59.5 ; 51.5 of this value is due to the processing times of the jobs and is called the processing time effect; 8 of this value is due to the PM visit duration and is called the PM effect. When we increase the cutting parameters $v$ and $f$ the processing time is decreased, whereas the PM index is increased. There is a trade-off between the processing time effect and the PM effect. Decreasing the processing times will increase the PM indices, which may result in a longer total completion time. 
Table 1. Experimental design factors.

\begin{tabular}{llcc}
\hline Factor & \multicolumn{1}{c}{ Definition } & Level 1 & Level 2 \\
\hline$\tau_{\mathrm{pm}}$ & PM duration & 30 & 90 \\
$A$ & PM function parameter & 10 & 20 \\
$B$ & PM function parameter & 30 & 45 \\
\hline
\end{tabular}

Table 2. Technical coefficients and parameters.

\begin{tabular}{lccl}
\hline$\alpha$ & 4.2 & $C_{\mathrm{O}}$ & 0.5 \\
$\beta$ & 1.65 & $C_{\mathrm{t}}$ & 1.25 \\
$\gamma$ & 1.2 & $S$ & $\mathrm{UN}[300,500]$ \\
$C$ & 56158018 & $D$ & $\mathrm{UN}[2.5,4]$ \\
$b$ & 0.9 & $L$ & $\mathrm{UN}[5,9]$ \\
$c$ & 0.78 & $d$ & $\mathrm{UN}[0.025,0.3]$ \\
$e$ & 0.65 & $H$ & 10 \\
$C_{\mathrm{m}}$ & 1.706 & $T$ & 2000 \\
$g$ & -1.54 & $k$ & 2.5 \\
$h$ & 1.04 & $C_{\mathrm{PM}}$ & 10 \\
$l$ & 0.32 & $C_{\mathrm{s}}$ & 211825000 \\
$t_{\mathrm{r}}$ & 1 & & \\
\hline
\end{tabular}

We used three experimental factors in our computations as listed in table 1, and each factor has two possible values. It is a $2^{3}$ full factorial design. Ten replications of each factorial setting are taken, resulting in 80 different randomly generated runs. The first experimental factor in table 1 is $\tau_{\mathrm{pm}}$, the PM visit duration. It is the time that the machine is not available during a PM visit. It strongly influences the total completion time of a given schedule, as discussed above. The other two experimental factors are $A$ and $B$, which are the coefficients for the PM index function. We generated 2000 jobs for each run. The parameters used to generate jobs are given in table 2.

Table 3 summarizes the minimum, average and maximum values of the completion time for each objective function at different levels of $\tau_{\mathrm{pm}}$. In this table, level 1 and level 2 of $\tau_{\mathrm{pm}}$ are represented by 1 and 2, respectively, where the results are averaged over 40 runs. On the other hand, the overall results over the 80 randomly generated runs are given in the 'Total' row. From table 3 we can see that the minimum average total completion time is achieved by the proposed $M+T+P M$ objective function. We can also see that, rather than minimizing processing times or minimizing PM indices of jobs independently, considering the machining, PM and tooling costs simultaneously improves the total completion times. This is due to the fact that, by considering these costs, we balance the processing time effect, the PM effect and the tool replacement effect on the total completion time objective. This means that decreasing tooling and/or PM costs necessitates longer processing times, whereas decreasing machining costs necessitates shorter processing times. We conclude that the total completion times for all settings are strongly affected by the $\tau_{\mathrm{pm}}$ level due to the analysis of variance (ANOVA) 


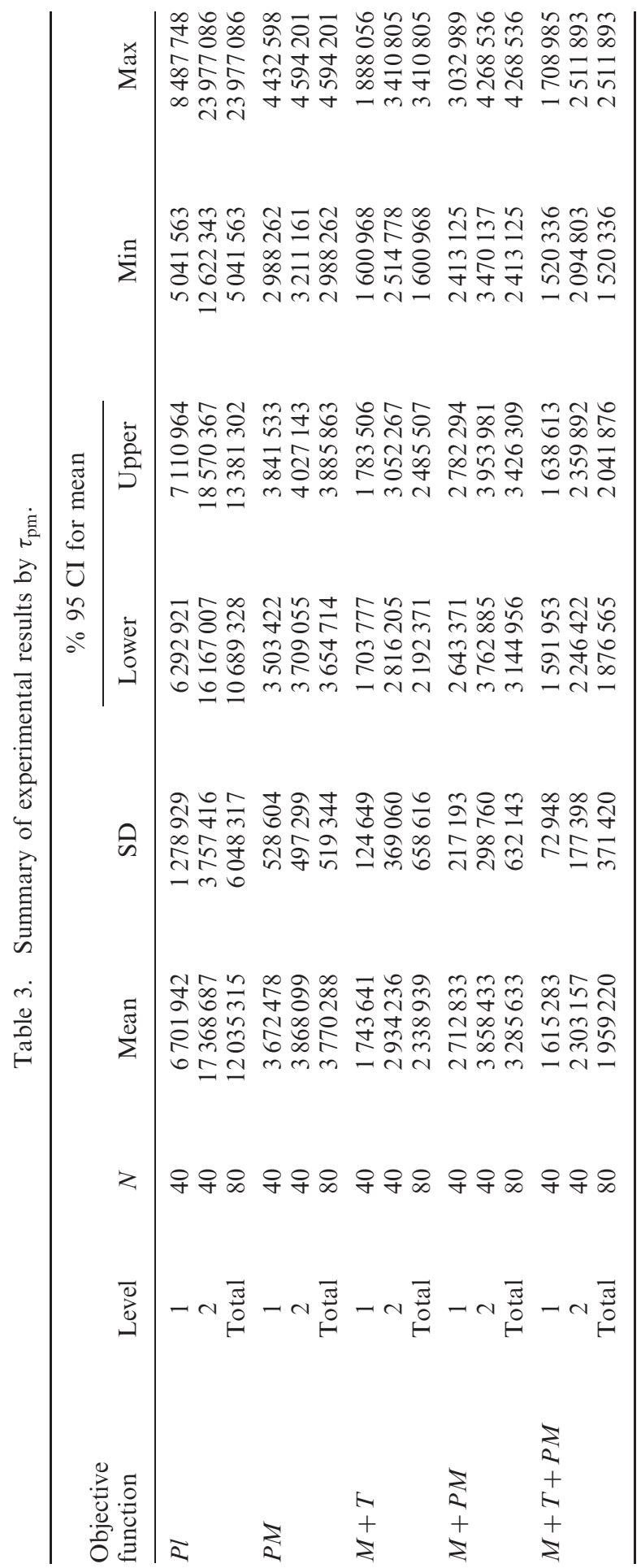


Table 4. ANOVA results with respect to $\tau_{\mathrm{pm}}$.

\begin{tabular}{lrc}
\hline & $F$ & Sig. \\
\hline$P l$ & 288.9 & 0.000 \\
$P M$ & 2.9 & 0.092 \\
$M+T$ & 373.7 & 0.000 \\
$M+P M$ & 384.8 & 0.000 \\
$M+T+P M$ & 514.4 & 0.000 \\
\hline
\end{tabular}

results, which can be seen in table 4. Our computational experiments indicate that considering the machining conditions in PM decisions not only influences the manufacturing costs but also affects the scheduling performance of a CNC machine.

\section{Concluding remarks}

In this paper we present a new condition-based maintenance approach for CNC turning machines. This approach aims to link the operating conditions decisions with maintenance decisions, which will improve maintenance performance and machine utilization. While making the PM decisions, a higher level problem in the decision making hierarchy, our model handles the tool replacement decisions, a lower level decision-making problem, using the failure replacement strategy. The developed model can be used as a PM planning tool for CMMS software. An important question in the current literature on condition-based maintenance is how to measure the performance degradation of machines. If degradation can be measured, then corrective maintenance activities can be performed before a failure occurs. We have established a mathematical relationship between the machining conditions (or production rate) and the PM needs of a machine. With the help of the proposed PM index we can change the maintenance strategy from the current mean-time-between-failure (MTBF) practices to a more intelligent technology that is based on the operating conditions of the CNC machine. Furthermore, we extend the classical SMOP formulation by adding a PM cost term to the objective function, which enables us to include maintenance considerations when making machining conditions decisions. We develop an algorithm to solve this new problem. Finally, we investigate the impact of considering different objective functions in the SMOP on the scheduling performance. As automated systems require effective maintenance management we can consider this study as an attempt to provide this.

Although our analysis has been performed for a specific PM index function which we have derived for a certain PM costing scenario, we believe that the results can easily be extended to a general type of convex decreasing PM index function that does not violate the geometric programming structure of SMOP. As a future research direction, we will consider the total completion time problem for a single CNC machine with controllable processing times where we have a machine availability constraint due to PM visits. 


\section{Acknowledgement}

The authors would like to thank the anonymous referees for their guidance in improving the contents of the paper.

\section{References}

Akturk, M.S. and Avci, S., Tool allocation and machining conditions optimization for CNC machines. Eur. J. Oper. Res., 1996, 94, 335-348.

Banjevic, D., Jardine, A.K.S., Makis, V. and Ennis, M., A control-limit policy and software for condition-based maintenance optimization. INFOR, 2001, 39, 32-50.

Bazaraa, M.S., Sherali, H.D. and Shetty, C.M., Nonlinear Programming: Theory and Algorithms, 1993 (Wiley: New York).

Cassady, C.R. and Kutanoglu, E., Minimizing job tardiness using integrated preventive maintenance planning and production scheduling. IIE Trans., 2003, 35, 503-513.

Fenton, R.G. and Joseph, N.D., The effect of the statistical nature of tool life on the economics of machining. Int. J. Mach. Tool Des. Res., 1993, 19, 43-48.

Gopalakrishnan, B. and Al-Khayyal, F., Machine parameter selection for turning with constraints: an analytical approach based on geometric programming. Int. J. Prod. Res., 1991, 29, 1897-1908.

Gorczyca, F.E., Application of Metal Cutting Theory, 1987 (Industrial Press: New York).

Gray, A.E., Seidman, A. and Stecke, K.E., A synthesis of decision models for tool management in automated manufacturing. Mgmt Sci., 1993, 39, 549-567.

Groover, M.P., Fundamentals of Modern Manufacturing: Materials, Processes and Systems, 2002 (Wiley: New York).

Higgins, L.R., Mobley, R.K. and Brautigam, D.P., Maintenance Engineering Handbook, 1995 (McGraw-Hill: New York).

Hitomi, K., Manufacturing Systems Engineering: A Unified Approach to Manufacturing Technology, Production Management and Industrial Economics, 1996 (Taylor and Francis: London).

Iakovou, E., Ip, C.M. and Koulamas, C., Optimal solutions for the machining economics problem with stochastically distributed tool life. Eur. J. Oper. Res., 1996, 92, 63-68.

Iakovou, E., Ip, C.M. and Koulamas, C., Throughput-dependent periodic maintenance policies for general production units. Ann. Oper. Res., 1999, 91, 41-47.

Jardine, A.K.S., editor, Optimizing condition based maintenance decisions, in Annual Reliability and Maintainability Symposium, 2002 (IEEE: New York).

Kaspi, M. and Shabtay, D., Optimization of the machining economics problem for a multistage transfer machine under failure, opportunistic and integrated replacement strategies. Int. J. Prod. Res., 2003, 41, 2229-2247.

Kelly, C.M., Mosier, C.T. and Mahmoodi, F., Impact of maintenance policies on the performance of manufacturing cells. Int. J. Prod. Res., 1997, 35, 767-787.

Malakooti, B. and Deviprasad, J., An interactive multiple criteria approach for parameter selection in metal cutting. Oper. Res., 1989, 37, 805-818.

Mitchell, J.S., The history of condition monitoring and condition based maintenance. Sound Vibr., 1999, 33, 21-28.

Nolden, C., Predictive maintenance. A route to zero unplanned downtime. Plant Engng, 1987, 41, 38-43.

Shabtay, D. and Kaspi, M., Optimization of the machining economics problem under the failure replacement strategy. Int. J. Prod. Econ., 2002, 80, 213-230.

Swanson, L., The impact of new production technologies on the maintenance function: an empirical study. Int. J. Prod. Res., 1999, 37, 849-869.

Wang, W., A model to determine the optimal critical level and the monitoring intervals in condition-based maintenance. Int. J. Prod. Res., 2000, 38, 1425-1436. 